\title{
Biometric Signature of Private Key by Reliable Iris Recognition Based on Flexible-ICA Algorithm
}

\author{
Aissa Boukhari, Salim Chitroub, Imen Bouraoui \\ Signal and Image Processing Laboratory, Telecommunication Department, \\ Electronics and Computer Science Faculty, University of Science and Technology of \\ Houari Boumedienne (USTHB), Algiers, Algeria \\ E-mail:aissaboukhari@yahoo.fr \\ Received September 29, 2011; revised November 7, 2011; accepted November 20, 2011
}

\begin{abstract}
The numerical world is under a fast development generating facilities and threats. The recommended solutions are especially the protection of information in all its states. The levels of protection show a discrepancy from an application to another; governmental, commercial or even cybercriminal. The infrastructure used in modern cryptography is based on public key cryptosystem. The problem is how to make safe the private key and to memorize it without difficulties and damages. This paper introduces a biometric solution of owner signature generating an encryption of the key using the iris recognition kept in a smart card. Several precautions were taken to guarantee the safety and the availability of the use of the private key. They are two essential goals to attest: the quality of the service and the robustness of suggested safety. Being the quality of the service, the used iris recognition is based on a new emerging method founded on Flexible-ICA algorithm. This method offers a better Equal Error rate compared to other methods previously used. This quality of recognition was also reinforced by an encoding of error using a flag and finally Reed Solomon encoder. For recommended safety, a scheme based on block encryption is used. The proposed scheme is Propagating $\mathrm{Ci}$ pher Block chaining which offers a very propagation of a high level of confusion and diffusion. Indeed, the robustness of this cryptographic process was studied by setting up strict criteria of safety.
\end{abstract}

Keywords: Image Processing, Cryptosystem, Public Key, Iris Recognition, Code Reed Solomon, Independent Component Analysis (ICA)

\section{Introduction}

The current world of the data security lived a very important jump. The scopes of application are the crucial factors requiring of the complex, robust and especially owners schemes.

Nowadays, the E-commerce, E-banking, E-voting, and so on became part of the daily people's life. However, the kind of cryptographic system used is of public keye.g. RSA [1,2].The problematic of the human being is divided in two parts. The first part is the robustness of the private key. The second part is the manner of storage. In the first part, the requirement implies that not only the private key satisfy the criteria's security of the conception of the public key cryptosystem, but also the length of the key. As example of RSA, a public key of 2048 bits, generated from the big primer numbers, is strongly recommended [3]. In the second part, some problems are born following the realization of the first part. Indeed, how could a human being remember such a large key e.g. 2048 bits?

Secure storage solutions have been proposed. The subscription in a file easily accessible was avoided [4]. Also, the passwords, that are typically short, are breakable and susceptible to the dictionaries attacks [4] in which the attacker tried several passwords lists to decrypt the cryptogram containing the private key.

In order to thwart these problems, we propose a biometric signature method using the irises. This will achieve three goals: Avoid the memorization of a short password. By the way, the owner confidentiality and the authentication of the users are assured by a specific system to each person. Finally, the no repudiation is related to the user's biometric features. No one can deny to have used his or her specific biometric mean [5].

In the literature, several considerable approaches of 
security using the biometric data were proposed. There are those that generate key's encryption directly from the biometric measures as Tomko et al. [6] using the fingerprints. Gohand Ngo [7] used a random projection of user's face as source of keys generation. In 2001 Monroses et al. [8] propose a combination of password with the user's voice. This last method has been compromised by the small entropy of the biometric key which is about 46 bits [8]. An approach using the irises was proposed by Hao et al. [9]. They used the smart card and a coding chain achieved around the Reed Solomon and Hamming encoder in order to procure a key of 140 bits.

Otherwise, the biometric systems have their own problems. For example the iris's features scan of the same person is almost always different e.g. from $10 \%$ to $20 \%$ according to [10]. The second problem is the irrevocability of the biometric signatures. Indeed, no one can store the biometric features in the free spaces. This proves to be dangerous because the attacker or the robber can take the complete user's identity.

In this work, we propose some solutions to these constraints. For the first constraint, we will use a new manner of recognition of iris. It is Flexible-ICA algorithm for reliable iris recognition. This method carried out recently by giving a FFR (False Features rejection) which tends towards zero.

Many researchers have worked on iris recognition including image databases, and human iris authentication process basically consists of four steps as follows: 1) iris segmentation, where the iris is localized and isolated from the noise due to sclera, pupil, eyelids and eyelashes; 2) normalization, where iris is mapped from rectangular representation to domain polar representation; 3) feature extraction, where a feature vector is formed which consists of the ordered sequence of the features extracted from the various representation of the iris images; 4 ) and matching, where the feature vectors are classified through different techniques such as Hamming Distance, weight vector and winner selection, dissimilarity function, etc. In our work, we first use Canny edge detection and Hough transform for iris localization. Then, the extracted iris region is normalized into a rectangular block with constant dimensions to account for imaging inconsistencies of Daugman's model. We apply Flexible-ICA algorithm to extract efficient feature vectors. Then, each iris feature vector is encoded into an iris code. Finally, a Hamming distance is used, for the matching process. We demonstrate our experimental results using two different subsets of CASIA-V3 iris image database and some mathematical criteria, in order to compare this technique against some other existing methods in order to assess its usefulness.

For the second constraint, we propose to use a joint solution. The first one was proposed in [11]. It is summarized in the creation of a flag vector to correct the features' iris. The use of the flag will be distinctly more efficient compared to the gotten results in [11]. In the previous works one used the Daugman's method [10] having a bigger FFR in relation to the Flexible-ICA method used in this paper. Also the complexity in the works of Sheikh Ziauddin and Matthew N. Dailey [11] is of 9600 bits for the features and 9600 bits for the masks. In this paper the proposed method based on the FlexibleICA algorithm gives a FRR about $4 \%$ for a complexity of 960 bits for the features. These results reduce strongly the rejected bits by the vector flag. Thus, there will be an effective features vector of about 960 bits on the one hand and on the other hand a low FRR. Indeed, in the jointed solution we propose to use an Error Encoder Correction (EEC) of Reed Solomon to correct the errors. With the good performances acquired by using FlexibleICA and the flag we will have an effective correction of error at $100 \%$.

To summarize the security of the private key, the user enrolls himself to create his own public data, namely: a vector flag and EEC code. These data will be store in a smart card. The same smart card will contain the encrypted private key with a cipher block cryptosystem using features of the iris like key encryption. The cipher block cryptosystem used is Propagating Cipher Block Chaining (PCBC). This mode of encryption was used in Kerberos protocol conceived by MIT (Massachusetts Institute of Technology) [12] which has given a strong authentication client/server. In this paper we will show the use of this mode PCBC assembled around standard AES with 256 bits key for encrypting the user's private key.

\section{Flexible-ICA for Features Extraction}

\subsection{Image Pre-Processing}

The iris is an annular part between the pupil (inner boundary) and the sclera (outer boundary). Therefore, a captured image cannot be expected to have only the iris part, it contains some non-useful part e.g. sclera, eyelid and pupil, therefore the iris region should be located in captured eye image, and normalized to polar array.

\subsection{Iris Localization}

Iris localization by definition means to isolate the actual iris region in a digital eye image by detecting the inner and outer boundaries of the iris. The eyelids and eyelashes normally occlude the upper and lower parts of the iris region. To detect the iris and pupil boundaries, 
Hough transform is used by involving Canny edge detection to generate an edge map. The gradients are biased in the vertical direction for the outer iris/sclera boundary while the vertical and horizontal ones are weightedequally for the inner iris/pupil boundary, as suggested in [13] and [14].

The Hough transform locates contours in an n-dimensional parameter space by examining whether they lie on curves of a specified shape. For the iris outer or pupillary boundaries and a set of recovered edge points $\left(x_{i}, y_{i}\right) i=1 \cdots n$, a Hough transform is defined by

$$
H\left(x_{c}, y_{c}, r\right)=\sum_{i=1}^{n} h\left(x_{i}, y_{i}, x_{c}, y_{c}, r\right)
$$

where $H\left(x_{c}, y_{c}, r\right)$ shows a circle through a point, the coordinates $x_{c}, y_{c}, r$ define a circle by the following equation

$$
x_{c}^{2}+y_{c}^{2}-r^{2}=0
$$

In the case of edge detection for iris boundaries, the above equation becomes

$$
\left(x_{i}-x_{c}\right)^{2}+\left(y_{i}-y_{c}\right)^{2}-r^{2}=0
$$

The eyelids are then isolated by first fitting a line to the upper and lower eyelid parts using a linear Hough transform. A second horizontal line is then drawn, which intersects with the first line at the iris edge that is closest to the pupil. The second horizontal line allows a maximum isolation of eyelid regions while a threes holding operation is used to isolate eyelashes.

\subsection{Iris Normalization}

Normalization refers to preparing a localized iris image for the feature extraction process. The process involves unwrapping iris image and converting it into its polar equivalent. It is carried out by using Daugman's Rubber sheet model $[15,16]$ as shown in Figure 1. The center of the pupil is considered as the reference point and a remapping formula is used to convert the points on the Cartesian scale to the polar scale.

The remapping of iris image $I_{(x, y)}$ from raw Cartesian coordinates to polar coordinates $(r, \theta)$ can be represented as

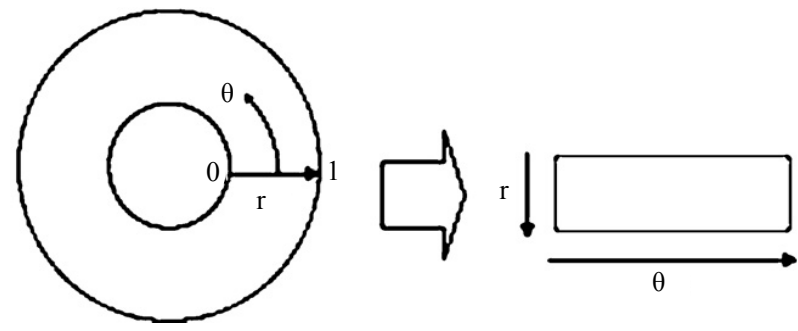

Figure 1. Daugman's rubber sheet model.

$$
I(x(r, \theta)), y(r, \theta) \rightarrow I(r, \theta)
$$

where $r$ is on the interval $[0,1]$ and $\theta$ is angle $[0,2 \pi]$, with

$$
\left\{\begin{array}{l}
x(r, \theta)=(1-r) x_{P}(\theta)+r x_{I}(\theta) \\
y(r, \theta)=(1-r) y_{P}(\theta)+r y_{I}(\theta)
\end{array}\right.
$$

where

$$
\left\{\begin{array}{l}
x_{P}(\theta)=O x_{P}(\theta)+r_{P}(\cos (\theta)) \\
y_{P}(\theta)=O y_{P}(\theta)+r_{P}(\sin (\theta))
\end{array}\right.
$$

And

$$
\left\{\begin{array}{l}
x_{I}(\theta)=O x_{I}(\theta)+r_{I}(\cos (\theta)) \\
y_{I}(\theta)=O y_{I}(\theta)+r_{I}(\sin (\theta))
\end{array}\right.
$$

The centre of the pupil is denoted by $\left(O x_{P}, O y_{p}\right)$ and $\left(O x_{I}, O y_{I}\right)$ is the center of the iris; $\mathrm{rP}$ is the radius of the pupil and $r_{I}$ is the radius of the iris; and $\left(x_{P}, y_{p}\right)$ and $\left(x_{I}, y_{I}\right)$ are the coordinates of points bordering the pupil's radius and iris' radius respectively along the direction $\theta$.

\subsection{Feature Extraction by ICA}

The iris has an interesting structure and presents rich texture information. The distinctive spatial characteristics of the human iris are available at a variety of scales [17]. As such, a well-known subspace analysis technique such as Independent Component Analysis (ICA) is used to capture local distinctive information in an iris and creates a set of compact features for an effective recognition task.

\subsubsection{Independent Component Analysis}

ICA represents a novel and powerful statistical method for subspace analysis, with applications in computational neuroscience and engineering. It consists of automatically identifying the underlying components in a given data set. It requires that at least as many simultaneously recorded mixtures as there are components and each mixture is a combination of components that are independent and nongaussian. However, like all methods, the success of ICA in a given application depends on the validity of the assumptions on which ICA is based and the results should be treated with caution. So, much theoretical work remains to be done on precisely how ICA fails when its assumptions, i.e. linear mixing and statistical independence, are severely violated $[18,19]$.

Generally, the most popular noising-free linear model of ICA is expressed as follows

$$
X=A S
$$


where $X$ is a vector variable, of dimension $N$, in which each variable is an observed signal mixture and $\mathrm{S}$ is a vector variable, of dimension $M$, in which each variable is a source signal. We assume that $N>M$. The mixing matrix A defines a linear transformation on $S$, which can usually be reversed in order to recover an estimate $U$ of $S$ from $X$, i.e.

$$
S \approx y=W X
$$

where the separating matrix $W=A^{-1}$ is the inverse of A. However, A is an unknown matrix and cannot therefore be used to find $W$. Instead, many iterative algorithms are used to approximate $W$ in order to optimize independence of S. In this paper, the Flexible-ICA algorithm [20] is deployed.

Since mutual information is the natural information-theoretic measure of the independence of random variables, it could be used as the criterion for finding the ICA transform. In this approach, which is an alternative to the model estimation approach, the ICA of a random vector $X$ is defined as an invertible transformation as in (9), where the matrix $W$ is determined so that the mutual information of the transformed components of $S$ is minimized.

Mutual information is a natural measure of the dependence between random variables. It can be interpreted by using the concept of differential entropy $H$ of a random vector y with density $f($.$) as follows [21]$

$$
H(y)=-\int f(y) \log f(y) \mathrm{d} y
$$

Entropy is considered as the coding length of the random variable $y_{i}, i=1 \cdots N$. In fact, it is defined as

$$
H\left(y_{i}\right)=-\sum_{i} P\left(y_{i}\right) \log P\left(y_{i}\right)
$$

However, mutual information $I$ between the $N$ (scalar) random variables $y_{i}, i=1 \cdots N \quad[22,23]$, is defined as

$$
I\left(y_{1}, y_{2}, \cdots, y_{N}\right)=\sum_{i}^{N} H\left(y_{i}\right)-H(y)
$$

Using the invertible linear transformation presented in (9). Mutual information [22], [21] is given by

$$
I\left(y_{1}, y_{2}, \cdots, y_{N}\right)=\sum_{i} H\left(y_{i}\right)-H(x)-\log |\operatorname{det} W|
$$

To search space of separating matrix or Stiefelmanifold $W$, let us consider that $y_{i}$ have been uncorrelated and have unit variance. This means

$$
E\left[y y^{T}\right]=W E\left[x x^{T}\right] W^{T}=I
$$

which implies

$$
\operatorname{det} I=1=\operatorname{det} W E\left[x x^{T}\right] W^{T}=\operatorname{det} W \operatorname{det} E\left[x x^{T}\right] \operatorname{det} W^{T}
$$

This implies that (det $W$ ) must be constant. In this case, the minimization of mutual information leads to the fol- lowing loss function

$$
L(W)=-\log p_{i}\left(y_{i}\right)
$$

The gradian of loss function (16) is given by

$$
\nabla L(W)=\frac{\partial L(W)}{\partial W}=\varphi(y) x^{T}
$$

where

$$
\varphi(y)=\left[\varphi_{1}\left(y_{1}\right), \cdots, \varphi_{N}\left(y_{N}\right)\right]^{T}
$$

and

$$
\varphi\left(y_{i}\right)=-\frac{\mathrm{d} \log p_{i}\left(y_{i}\right)}{\mathrm{d} y_{i}}
$$

The natural Reimannian gradient in Stiefel Manifold was calculated by [24] and it can be written as follows

$$
\begin{aligned}
\bar{\nabla} L(W) & =\nabla L(W)-W[\nabla L(W)]^{T} W \\
& =\varphi(y) x^{T}-y \varphi^{T}(y) W
\end{aligned}
$$

With this, the learning algorithm for $W$ takes the form $[25,26]$

$$
\Delta W=-\eta \bar{\nabla} L(W)=\eta\left[y \varphi^{T}(y) W-\varphi(y) x^{T}\right]
$$

where $\eta$ is a learning rate (small positive constant) and $\varphi(y)$ is non-linear function, noted by

$$
\varphi(y)=\frac{1}{a_{1}} \log \left(\cosh \left(a_{1} y\right)\right)
$$

where $1<a_{1}<2$ is some suitable constant.

In the learning process, the increment $\Delta W$ should satisfy the constraint

$$
\Delta W W^{T}+W \Delta W^{T}=0
$$

\subsubsection{Feature Extraction}

Image representations are often based on discrete linear transformations of the observed data. Consider a blackand-white image whose gray-scale value at the pixel indexed by $x$ and $y$, denoted by $I(x, y)$. Many basic models in image processing express the image $I(x, y)$ as a linear superposition of some features or basis functions $a_{i}(x, y)$, that is

$$
I(x, y)=\sum_{i=1}^{M} a_{i}(x, y) s_{i}
$$

where $s_{i}$ are feature coefficients. These basis functions, $a_{i}(x, y)$, are able to capture the inherent structure of the iris texture. This, particularity allows us to apply ICA and thus create a set of compact features for an effective recognition task. Alternatively, we can just collect all the pixel values in a single vector $X$, in which case we can express the representation as in (8) for ICA model. We assume here that the number of transformed components 
is equal to the number of observed variables. This type of a linear superposition model gives a useful description on a low level support where we can ignore such higherlevel nonlinear phenomena such as occlusions. For the sake of simplicity, let us restrict ourselves here to the simple case where the variables $a_{i}(x, y)$ form an invertible linear system, that is, the matrix $A$ is square. Then we can invert the system as:

$$
s_{i}=\sum_{x, y} w_{i}(x, y) I(x, y)
$$

where the $w_{i}$ denote the inverse filters of ICA.

In practice, we cannot model a whole image using the model in (24). Rather, we apply it on image patches or windows [18]. Thus we partition the image into patches of $n \times n$ pixels and model the patches with the model in (24). However, care must then be taken to avoid border effects.

Before extracting the iris features, we note that the ICA application is greatly simplified if the vector $X$ of all iris images is first whitened or sphered. There are two common pre-processing steps. The first step is to center the images as, $X=X-E\{X\}$ in order to make their local mean equal 0 . The next step is to apply a whitening transform B to the data such that

$$
B=D^{-1 / 2} E^{T}
$$

with $E$ corresponds to the eigenvectors of the covariance matrix of $X$ and the diagonal matrix $D$ contains the related eigenvalues. The whitening process helps to uncorrelate the data so that Principal Component Analysis (PCA) can work with a unit variance. The whitened data are used as the input for the Flexible-ICA algorithm [20], demonstrated above, which computes a set of basis vector, $w_{i}$ from a set of iris images, and the images are projected into the compressed subspace to obtain a set of coefficients, $s_{i}$. New test images are then matched to these known coefficients by projecting them onto the basis vectors and finding the closest coefficients in the subspace.

\subsection{Matching}

It is very important to present the obtained feature vector in a binary code because it is easier to determine the difference between two binary code-words than between two number vectors. In fact, Boolean vectors are always easier to compare and to manipulate. We have applied a Hamming Distance matching algorithm for the recognition of two samples. It is basically an exclusive OR (XOR) function between two bit patterns. Hamming Distance is a measure, which delineates the differences of iris codes. Every bit of a presented iris code is compared to the every bit of referenced iris code, if the two bits are the same, e.g. two 1's or two 0's, the system assigns a value " 0 " to that comparison and if the two bits are different, the system assigns a value " 1 " to that comparison. The formula for iris matching is shown as follows

$$
H D=\frac{1}{N} \sum P_{i} \oplus Q_{i}
$$

where $N$ is the dimension of feature vector, $P_{i}$ is the $i^{t h-}$ component of the presented feature vector, while $Q_{i}$ is the $i^{\text {th }}$ component of the referenced feature vector.

\section{Biometric Signature of Private Key Process}

\subsection{User Enrollment}

In this section one exposes the enrollment phase of the owner user data. The data are: the flag vector, the EEC code resulting from Reed Solomon encoding of the reliable iris features. The Figure 2 shows this enrollment phase and the flag creation learning.

We note that the public key, mentioned in this figure, is delivered to a certificate authority in order to generate the certificate that contains their relative data. This part won't be studied in this paper. Our goal is to secure the private key with an owner signature.

\subsubsection{Vector Flag Generation}

In practice, some iris areas of the same person are reliable than others. This is due to the pupil-irises boundary and the irises-sclera boundary. The imperfection of detection is present in the inner and outer circles. Because of these difficulties, a user, wanting to be enrolled, presents $n$ iris scan. In our case of experimentation $n=3$.

For each iris scan one generates a template of 960 bits of features. To create a single unify iris template $T$, the flag is used. Indeed, to create the flag we detect in the template iris scan the reliable bits which correspond to the identical bits along $n$ templates. To these positions one assigns the value " 1 " for the vector flag if not the assigned value is " 0 ". In this way the vector flag F was build with 960 bits.

On the whole of the reliable bits, we take the first 523 bits. Finally the vector flag generated $F$ is stored in a smart card. The following gives an example for 3 templates of iris.

$\left.\begin{array}{|l}1010100111010101 \\ 1010000101010100 \\ 1010100111010100\end{array}\right\} \begin{aligned} & \text { The flag vector } \mathrm{F} \text { is: } \\ & 1111011101111110 \\ & \text { The template unique } \mathrm{T} \text { is: } \\ & 1010001101010\end{aligned}$




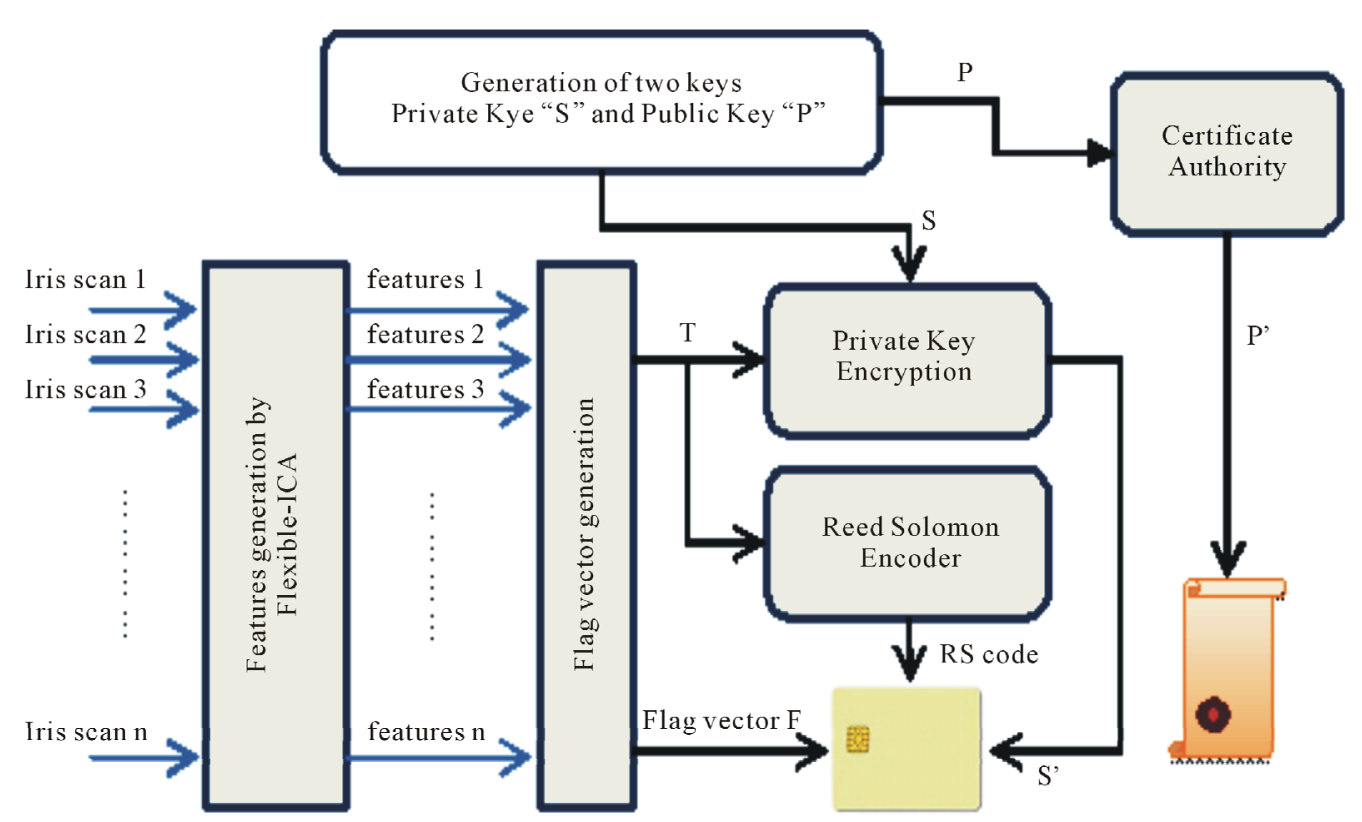

Figure 2. Biometric signature of private key process.

\subsubsection{Template Encoding Using Reed Solomon Encoder}

\subsubsection{Reed Solomon Encoder}

Reed Solomon encoder, noted $R S(N, K)$ [27] is a cyclic encoder allowing the detection and the correction of the errors per payload.This encoder is represented on the Galois field of $G F\left(2^{m}\right)$ and consequently it transforms a word of $N$ symbols of $m$ bits by adding $R S=N-K$ symbols of redundancies.

The length of the code word checks the following equation [28]:

$$
N=\left(2^{m}-1\right) \text { and } m \geq 3
$$

The capacity of correction Reed Solomon encoder is related to the minimal distance within the meaning of Hamming. In other word, the smallest distance $d_{\text {min }}=N-K+1$ between two distinct code words from the code.

This shows that the encoder can correct $t=\frac{\left(d_{\min }-1\right)}{2}$ symbols of $m$ bits.

\subsubsection{The Template Encoding}

During the enrollment one uses a flag vector that was generated to select features of a common template called $T$. This template was the result of the application of the flag vector. And finally the first 523 bits were selected.

In this phase, a Reed code is generated by the encoding $T$. In order to carry out this process, in Reed Solomon encoding, important parameters will be given, namely: $N$,
$K$ and $t$.

In our experiment, the length of the RS code is like that adopted in [29].

This work followed the results found by Sim et al. [29] in the recognition and the reduction of FRR from $26 \%$ to $2,9 \%$. In this paper we propose the use of the FlexibleICAmethod which with its low level competitor FFR with all the old methods. Like summary, the parameters are: $N=2^{m}-1=1023$ bits, $t=250$ bits, and $K=523$ bits. At the end of this phase, the RS code is recorded in the smart card. See the Figure 2.

\subsection{Private Key Encryption}

In this second phase, the user's smart card will receive the essential data that is the encrypted private key. The process of the private key encryption follows the scheme represented in Figure 3. In this figure one notices that the first 512 bits of the template $T$ have been used like encryption key.

The encryption scheme is a Propagating cipher block chaining (PCBC) gone up around the standard AES (Advanced Encrypted Standard) of 256 bits key [25,26]. In our work we use two initialization vectors $V I_{1}$ and $V I_{2}$ of 128 bits each taken from the two first 128 bits of the template. As unique key of the blocks, we will use the 256 bits that follow the initialization vectors. The plain text, in our case is the private key, will be shared in blocks of 128 bits each for the encryption process.

The personalization of the PCBC scheme as using two initialization vectors is justified as follow: The first $V I_{1}$ vector is used classically to initialize the encryption of 


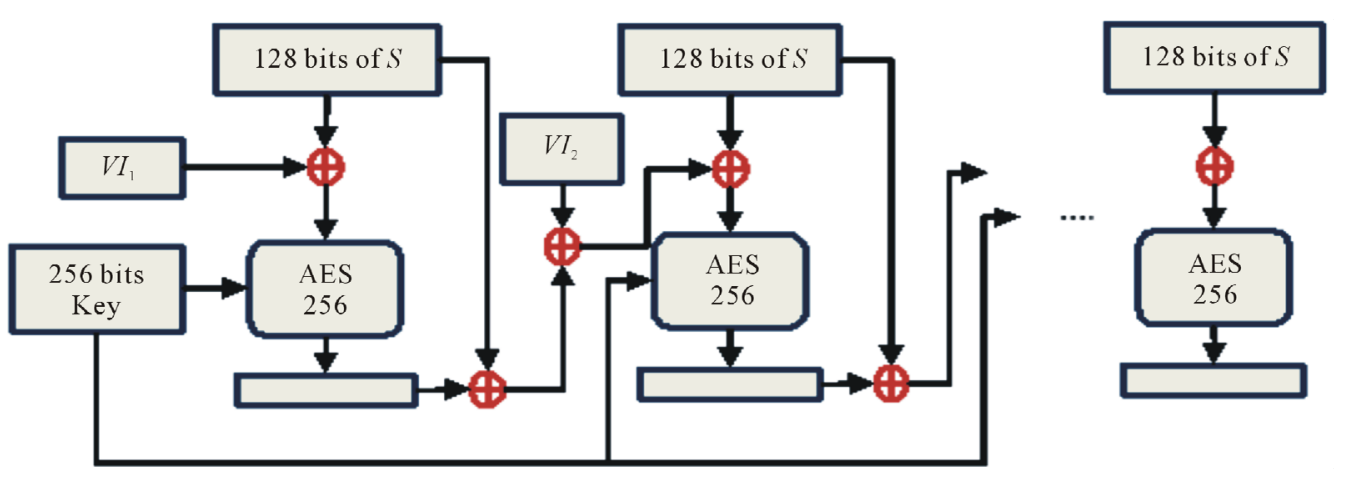

Figure 3. Private key encryption scheme.

the first plain block of 128 bits. The second initialization vector $V I_{2}$ will be used to increase confusion in the propagating phenomena. The introduction of $V I_{2}$ will eliminate all trace on the first plain block aimed for any possible attack. It will be added to each exit AES bloc.

If we note $C_{i}$ and $P_{i}$, respectively cryptogram and plain text of the $i^{\text {th }}$ block of the PCBC scheme and AES_ENC and AES_DEC the encryption and decryption functions of the blocks gone up around the AES standard of 256 bits key, we will have:

$$
\begin{aligned}
& \left\{\begin{array}{l}
C_{i}=A E S_{-} E N C\left(P_{i} \oplus P_{i-1} \oplus C_{i-1}\right) \oplus V I_{2} \\
P_{i}=A E S_{-} D E C\left(C_{i} \oplus V I_{2}\right) \oplus P_{i-1} \oplus C_{i-1}
\end{array}\right. \\
& \text { and } P_{0} \oplus C_{0}=V I_{1}
\end{aligned}
$$

At the end of the encryption process of the private key $S$, we concatenate the exits of every block to carry out the final cryptogram. The encrypted private key is noted $S^{\prime}$. At this moment the user's smart card will receive $S^{\prime}$ as final data.

\subsection{Private Key Decryption and Use}

In this section one gives the process to be carried out so that a user can exploit his private key. Indeed, with an iris scanner, a user allows to be identified. Thus a template is taken. The smart card containing the useful information will be used to extract the encrypted private key. The Figure 4 presents an example of the use of the smartcard.

The algorithm of decrypting the cryptogram $S^{\prime}$ hiding the private key $S$ is as follows:

- Application of the vector flag $F$, being in the smart card, on the template in order to withdraw the reliable features $T$;

- The features vector $T$ undergoes a correction by the code Reed Solomon and the RS codes which is in the smart card in order to produce a new vector $T^{\prime}$;

- Selection the first 523 bits from the vector $T^{\prime}$;

- Reconstitution of the initialization vectors $V I_{2}$ et $V I_{2}$ from the two firsts blocks of 128 bits of $T^{\prime}$, and create a 256 bits AES key for decryption from the remaining bits of $T^{\prime}$ $S$

Decrypting the cryptogram $S^{\prime}$ to have the private key

\section{Experimental Results: Evaluation and Discussion}

This section deals with the proposed biometric signature for private key, by evaluating the performance of Flexible-ICA algorithms for features extraction and their computation complexities and costs.

In order to compare the performance and accuracy of the used method against the iris recognition methods used in the literature relative to security by iris. So the evaluation focused two goals: quality of service and security level. In the first one, we studded the better level of the recognition given by the Flexible-ICA algorithm compared to the others methods. In the second one, we studded the security level of the encryption process. Then we showed how the encryption scheme are given a strong protection to the private key and taken solution from iris recognition algorithm.

To perform these experiences we used CASIA-IrisV3

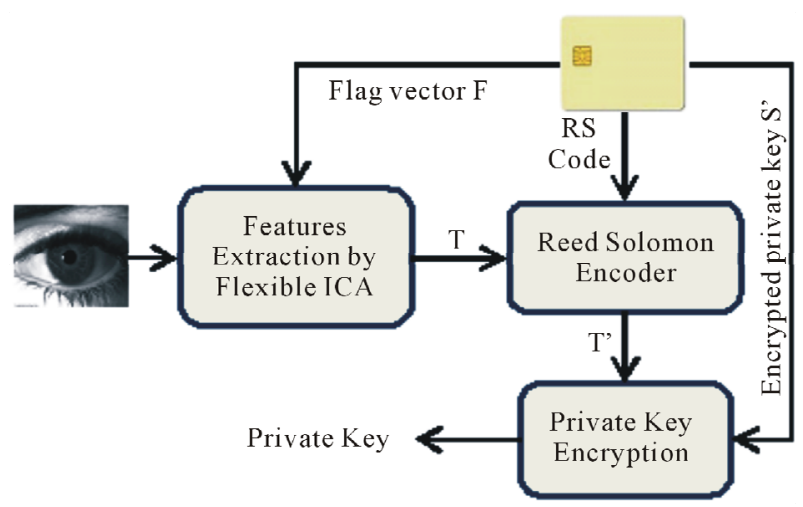

Figure 4. Theuse of smart card and decrypt the encrypted private key. 
includes three subsets which are labelled as CASIAIrisV3-Interval, CASIA-IrisV3-Lamp, and CASIA-IrisV3Twins [30]. All of the algorithms are implemented in MATLAB 7.4 and executed on the same computer (Intel ${ }^{\circledR}$ Pentium ${ }^{\circledR}$ T2080 Dual-Core 1.73 GHZ CPU, 1024 M RAM). All of the experiments are completed under the same conditions and environment.

\subsection{Quality of Service Evaluation}

\subsubsection{Features Extraction Experience}

To extract and evaluate results obtained with flexibleICA algorithm, we have used an ensemble of pre-processed image simples of size of $32 \times 240$. Each iris image should be localised by detecting its inner and outer boundary and its eyelids and eyelashes, unwrapped and converted into its polar equivalent where a number of data points are selected along each radial line and this is defined as the radial resolution and the number of radial lines going around the iris region is defined as the angular resolution. Then a histogram stretching method was used to obtain a well distributed iris images. Figure 5 gives an example of an iris sample of each subset with its pre-processing steps.

This ensemble contain1530respectively of CASIAIrisV3-Interval and CASIA-IrisV3-Lamp used features extraction process which consists of determining $S_{i}$ and $w_{i}(x, y)$ represented in (25). See Figure 6.

In this experience based on the Flexible-ICA, we use capture of class i.e. 306 or 228 images. These images were partitioned into 10,000 patches of $n \times n$ pixels randomly taken from the pre-processed images and then normalized to columns of $n^{2} \times 1$ and finally held into matrix of size $n^{2} \times 10,000$.

To calculate the separated matrix $W, X$ was projected in stifled manifold $W\left(R \times n^{2}\right)$ in order to obtain features vector $S$. The encoding method of iris in binary format is to assign values " 0 " and " 1 " like

$$
Q\left(S_{i}\right)=\left\{\begin{array}{l}
1 \text { if } S_{i}>0 \\
0 \text { if } S_{i} \leq 0
\end{array}\right.
$$

Finally, to compare irises, the Hamming distance was used.

\subsubsection{Evaluation Criteria}

To evaluate the features extraction based on FlexibleICA and compare it to others methods, we have used the ROC (Receiver Operating Characteristics') curve and EER (Equal Error Rate).

The ROC curve is the false acceptance rate (FAR) versus false rejection rate (FRR). The first one is the probability of accepting an imposter as an authorized subject.
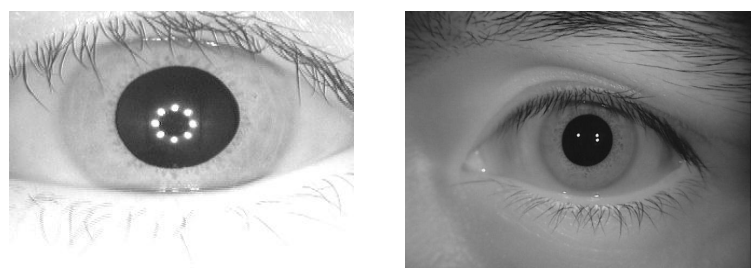

(a)
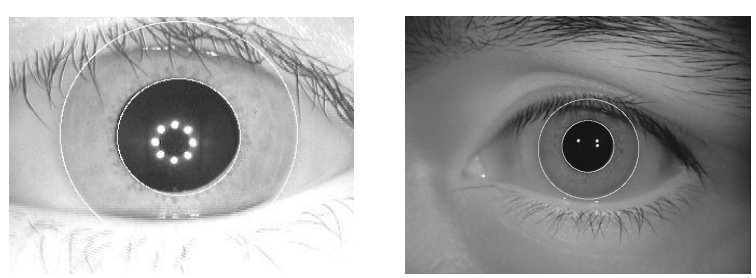

(b)
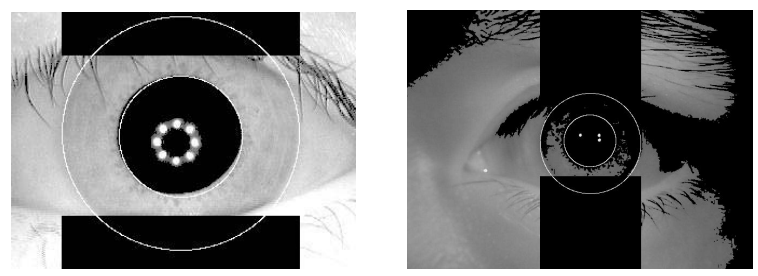

(c)
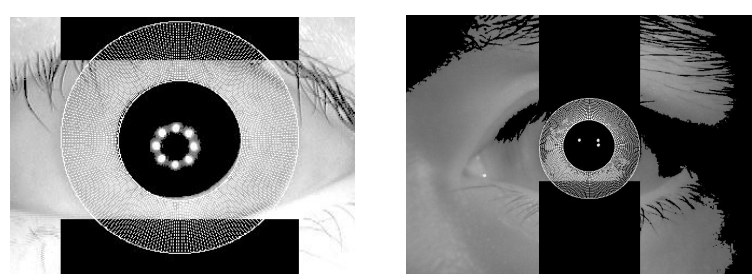

(d)
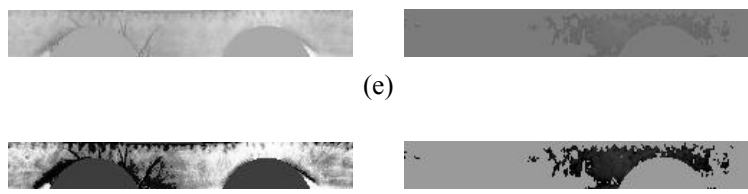

(e)

(f)

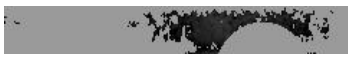

Figure 5. Irisimage pre-processing steps of a sample of each subset of CASIA Iris database, CASIA V3-Interval and CASIA V3-Lamp (left and right), (a) original iris; (b) iris localization; (c) eyelash and eyelids detection; (d) unwrapped iris with a radial resolution of 32 pixels and angular resolution of 240 pixels; (e) normalized; and (f) enhanced iris.

The second one is the probability that an authorized subject being incorrectly rejected. The deal FAR versus FRR curve is a horizontally straight line with zero false rejection rates. So, the EER is the point were FRR equal to FAR in value. The smaller EER is the better the algorithm.

These criteria were used to prove the level of quality of service given by the method based on Flexible-ICA compared to the others. Also the accuracy, features vector 


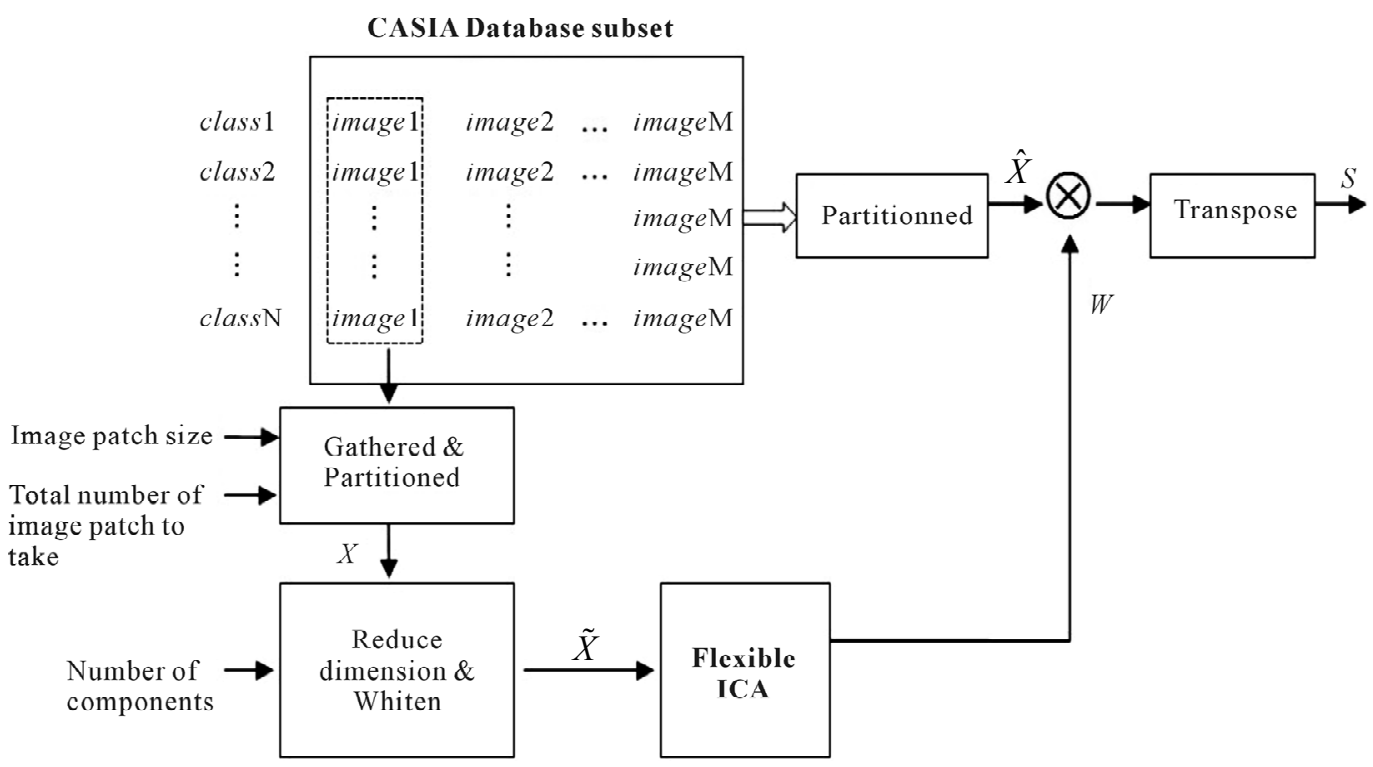

Figure 6. Block diagram of feature extraction process.

size and complexity are given in the next section.

\subsubsection{Results Study}

For assess of the Flexible-ICA algorithm in its recognition, each iris is compared to all other irises at intra or inter class of CASIA used subset. A total of 49,725 and 34,086 comparisons were executed respectively in CASIA-V3-Interval and CASIA-V3-Lamp.

Figure 7 shows the large distribution of the distance between the intra and inter class. Figure 7 reveals the variation of visible illumination makes the distribution of distance of CASIA-V3-Lamp is larger than the intra class distance distribution of CASIA-V3-interal. This is caused by bad results of phase localization and normalization.

To evaluate the EERs, Table 1 shows results for $\mathrm{R}=$ $\{56,40,32,24,20,16,12,10,8\}$ and image patches size of $16 \times 16$ or $8 \times 8$ pixels. We see that CASIA-V3-interval gives EERs lower than $0.2 \%$ because of its good quality resulting in extremely clear iris textures details. Like general remark, we see that ERRs increase when ICA coefficients decrease but when the information is strongly affected by noise according some coefficients the performance does not always decrease with reduction of ICA coefficients.

This observation is studded by results obtained with CASIA-V3-Lamp. This is the same remark like accuracy showed in Figure 6. To compare our results to others in the next section we take the better value in Table 1 .

\subsubsection{Quality Service Study}

In this section we present a comparison between three methods, used in the literature, like security based on iris
Table 1. Performance evaluation according to numbers of independent component and image patches sizes of CASIA V3-Interval and CASIA V3-Lamp.

\begin{tabular}{|c|c|c|c|c|c|}
\hline \multirow{2}{*}{\multicolumn{2}{|c|}{$\begin{array}{l}\text { Database } \\
\text { Win. size }\end{array}$}} & \multicolumn{2}{|c|}{ CASIA-V3-Interval } & \multicolumn{2}{|c|}{ CASIA-V3-Lamp } \\
\hline & & $8 \times 8$ & $16 \times 16$ & $8 \times 8$ & $16 \times 16$ \\
\hline \multirow{9}{*}{ ICs } & 56 & $0.10 \%$ & $0.03 \%$ & $15.69 \%$ & $12.48 \%$ \\
\hline & 40 & $0.03 \%$ & $0.10 \%$ & $16.89 \%$ & $12.34 \%$ \\
\hline & 32 & $0.16 \%$ & $0.03 \%$ & $16.88 \%$ & $15.54 \%$ \\
\hline & 24 & $0.10 \%$ & $0.34 \%$ & $16.22 \%$ & $13.62 \%$ \\
\hline & 20 & $0.14 \%$ & $0.86 \%$ & $16.19 \%$ & $18.09 \%$ \\
\hline & 16 & $0.04 \%$ & $0.45 \%$ & $16.82 \%$ & $17.08 \%$ \\
\hline & 12 & $0.16 \%$ & $3.95 \%$ & $19.93 \%$ & $20.83 \%$ \\
\hline & 10 & $0.13 \%$ & $3.72 \%$ & $16.76 \%$ & $19.28 \%$ \\
\hline & 8 & $0.13 \%$ & $2.25 \%$ & $18.34 \%$ & $23.96 \%$ \\
\hline
\end{tabular}

features. These methods are: Daugman [16,31], Ma et al. [32] and Tan et al. [33] using the CASIA-V3-interval iris image database [30]. So, we only analyze and compare the accuracy, efficiency and computational complexity.

Daugman represent the local shape of the iris details by phase information and projected each small local region onto bank of Gabor filters, then he quantize the resulting phase, denoted by complex valued coefficients, to one of the four quadrants in the complex plane. The dimensionality of the features vector is 2048 components.

Ma et al. method [32] constructs a set of intensity signals to contain the most important details of the iris and makes use of stable and reliable local variations of the 


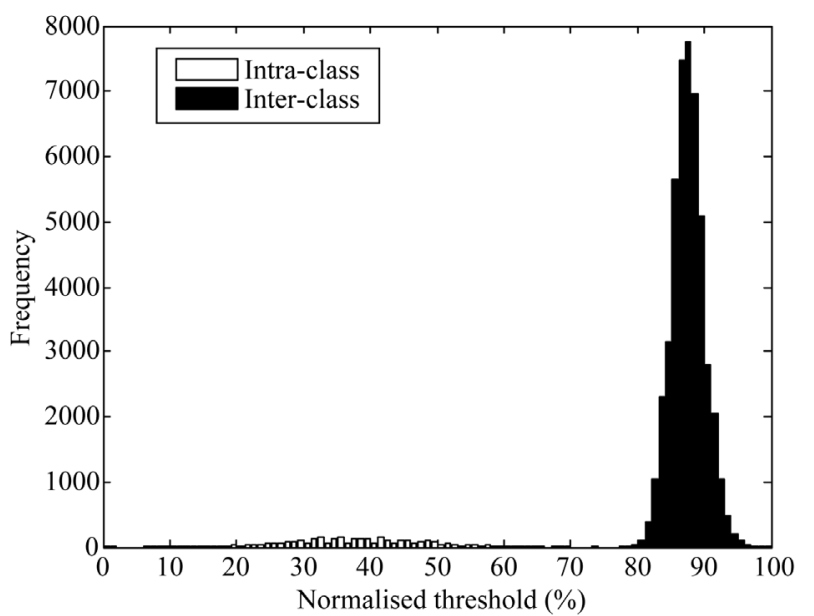

(a)

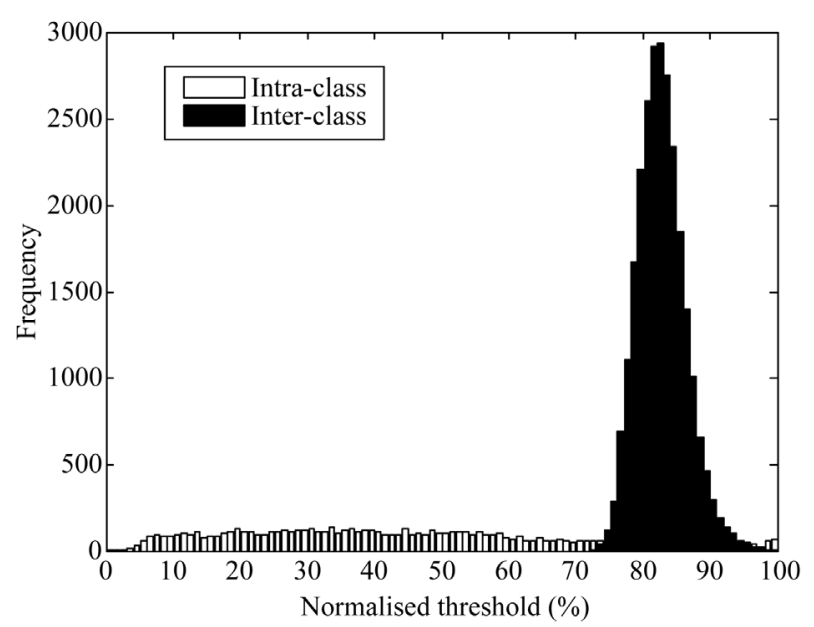

(b)

Figure 7. Results of intra-class and inter-class distributions of (a) CASIA V3-Interval and (b) CASIA V3-Lamp.

intensity signals as features, their method contains about 660 components, this is because that their method only records the position of local sharp variations as features and contains less redundant information.

In [33], Tan et al. utilize multichannel spatial filters to extract texture features of the iris within a wider frequency range, this indicates that the extracted features are more discriminating, they extract local characteristics of the iris from the viewpoint of texture analysis, and the dimensionality of the feature vector is 1600 components. Figure 8 give the ROC curve of such verification algorithm.

From the results shown in Figure 8, we can see that the flexible-ICA method has the best performance, followed by both Ma et al. and Daugman methods which are slightly better than the method of Tan et al. So, this method is based on flexible-ICA algorithm which extracts global features in pre-processing step that reduces dimensions for obtaining ICA components for iris; ICA explores independent components of fine iris features. These components of ICA are statistically independent, which reflect iris detail information (such as freckles, coronas, strips, furrows, crypts, and so on) change, whose distribution indicates iris individual difference for each class. So, the local basis images obtained with ICA can lead to more precise representations.

Since ICA reduces significantly the size of iris code, this leads to decrease of processing time. Table 2 shows that our method consumes less time than others, followed by both Tan and Ma methods which are based on 1-D signal analysis. However, Daugman method involves 2-D mathematical operation.

These comparisons indicate that the used algorithm has an effective and emerging performance in iris recognition. This remark is all in concordance with the quality of service including the best recognition. In security field it is not be able to accept any mistakes in the user recognition because the transaction or the use of the private key was corrupted.

\subsection{Security Analysis}

In this section we study how strong is our scheme to protect any private key stored in smart card.

Table 2. Performance comparison of the algorithms.

\begin{tabular}{cccc}
\hline Methods & $\begin{array}{c}\text { Feature vector } \\
\text { size (bit/image) }\end{array}$ & Performance (\%) $\begin{array}{c}\text { Computational } \\
\text { Complexity (ms) }\end{array}$ \\
\hline Daugman [9] & 2048 & 0.08 & 285 \\
Ma et al. [16] & 660 & 0.07 & 95 \\
Tan et al. [15] & 1600 & 0.48 & 80.3 \\
Proposed ICA & 960 & 0.04 & 31.2 \\
\hline
\end{tabular}

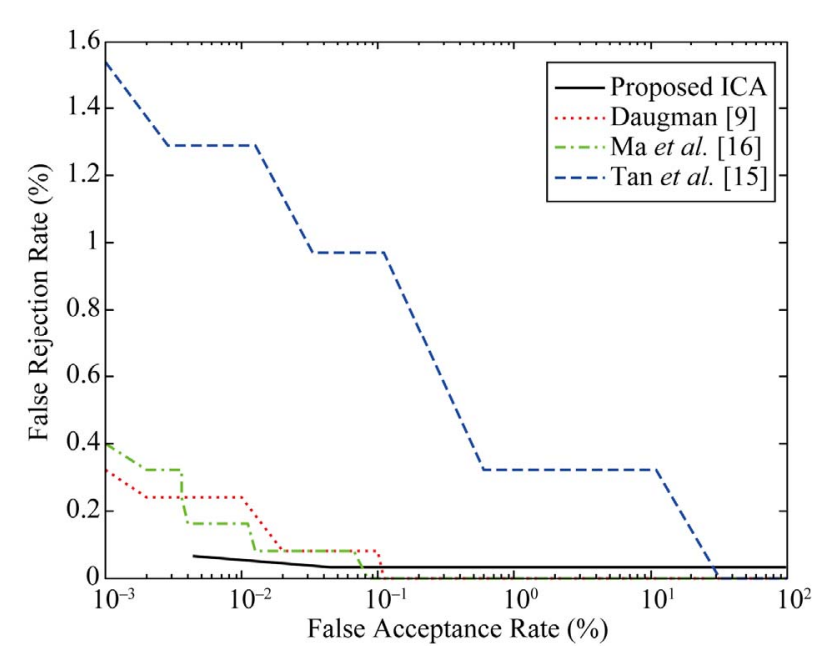

Figure 8. Comparison of ROC curves. 


\subsubsection{Iris Key Space Analysis}

If an attacker try to break our key used in encryption of private key, he would be systematically search the 523 bits key space for the correct iris code. So, we see that as long as, the iris code is kipped private and the brute force or more sophistical attack on the cryptosystem used the iris code is the best an attacker can do. In this focus, we consider that the powerful attacker who has complete knowledge on the correlation present between iris template space as well as complete knowledge of the Reed Solomon codeword space.

In the literature Daugman [10] has estimated the degree of freedom in iris template to be 249 bits. It means that these 249 bits are sufficient to reconstruct a valid iris template for one person, and likewise, are capable of representing the eyes of more than $2^{249}$ unique individuals.

In our case, if an attacker has complete knowledge of the structure of the iris template space, he could simply search 249 bits iris code. At each time a valid iris template for an individual is checked the correctness from Reed Solomon codeword. So this codeword can, in the worse correction, recovery 250 bits or the iris code used by the cryptosystem is equal to 523 bits. Then the attacker by all that, he has to complete 24 bits.

To estimate the complexity attack time we assumed that the time recovery of any code bit search is about 1 second then we have

$$
\text { Time BFA }=\left(2^{249+24} \times \text { Time RS }\right) \text { seconds }
$$

where, Time BFA is the time brute force attack and Time RS the time Reed Solomon recovery. Well, it is long time to do a best attack like this!

\subsubsection{Avalanche Effect}

The avalanche effect is the desirable property of cryptosystem. It means that if an input changed slightly e.g. flipping a single bit, the out put changes significantly e.g. a half of out bits flip. So, in our work, we use an AES256 bit like a bloc cipher system, then the avalanche effect is obtained from the security of this standard [34].

Since we use a Propagating Cipher bloc Chaining (PCBC), if a bit changed in $i^{\text {th }}$ bloc all flowed block changed for more than half of bits. It could arrive to change completely. For example at $i=1$ (first block), if the private key $S$, subject to encryption, changed at any bit from the first bloc of 128 bits the encrypted form of $S^{\prime}$ would change at most completely. This property is guaranteed by the propagating phenomena and this change from bloc to other gives a best avalanche effect.

\subsubsection{Confusion and Diffusion}

Like avalanche effect, these two properties guaranteed a statistical security means. Indeed, confusion is to avoid any relationship between key and cipher text and diffusion is to illuminate any redundancy in the plain text by dissipate it in the statistic of cipher text.

At each bloc, of the used PCBC, the confusion and diffusion are guaranteed systematically by AES-256 bit in first. Then each block of 128 bits of private key $S$ undergone if own appropriate transformations. However, our PCBC is mad by using two initialization vector vectors $V I_{1}$ and $V I_{2}$. The first one mad a complete transformation in the first block of $S$ before the encryption process. Also the second block, of $S$, accepts its own complete transformation by the second initialization vector $V I_{2}$ and the XOR of the first block and its encryption form.

The phenomena of propagating use for the rest of block a transformation of their block of $S$ the XOR of the plain and its encryption form of the previous block. All those give transformed plain bloc to each block of used AES. This is a guaranty of higher confusion and diffusion mad in the private key before and after the encryption process.

\section{Conclusions and Future Works}

In this paper we have given a complete system for encrypt and secure the private key of any public key infrastructure. Our contribution is to avoid any weak secure scheme previously proposed to a secure storage of private key. Our scheme is based on the use of biometric signature by reliable iris recognition. The originality is in the use of the Flexible-ICA for feature extraction with partition of iris images into patches, and hamming distance for matching. Two iris image subsets of CASIA iris V3 database have been used to evaluate the performance of our system. Flexible-ICA algorithm, which improves the quality of separation introducing a better density matching and allows a faster learning, has been adopted for computing the ICs.

Best results have been obtained. In the first, the quality of recognition, given by the way a high quality of service to recovery the private key without any error in the key encryption. To eliminate any probability of error, a joint flag and Reed Solomon encoder have used. Secondly, the proposed scheme has been evaluated to prove its robustness.

Like future works, we propose to use the Noisy-ICA algorithm for features extraction. This method avoids any problems made by the multiplicative and additive noise in iris scan.

\section{References}

[1] W. Stallings, "Cryptography and Network Security: Prin- 
ciples and Practice," 3rd Edition, Prentice Hall, Saddle River, 2003.

[2] R. F. Churchhouse, "Codes and Ciphers," Cambridge University Press, Cambridge, 2004.

[3] C. Paar and J. Pelzl, "Understanding Cryptography," Springer-Verlag, Berlin, Heidelberg, 2010, p. 173.

[4] B. Schneier, "Applied Cryptography-Protocols, Algorithms, and Source Code in C," 2nd Edition, John Wiley \& Sons, Inc., New York, 1996.

[5] D. R. Stinson, "Cryptography: Theory and Practice," 2nd Edition, CRC Press, Boca Raton, 2002.

[6] G. Tomko, C. Soutar and G. Schmidt, "Biometric Controlled Key Generation," United States Patent No. 5680460, 1997.

[7] A. Goh and D. Ngo, "Computation of Cryptographic Keys from Face Biometrics," Proceedings of International Conference on Communications and Multimedia Security, Torino, 2-3 October 2003, pp. 1-13.

[8] F. Monrose, M. K. Reiter, Q. Li and S. Wetzel, "Cryptographic Key Generation from Voice," Proceedings of the IEEE Symposium on Security and Privacy, Oakland, 1416 May 2001, pp. 202-213.

[9] F. Hao, R. Anderson and J. Daugman, "Combining Crypto with Biometrics Effectively," IEEE Transactions on Computers, Vol. 55, No. 9, 2006, pp. 1081-1088. doi:10.1109/TC.2006.138

[10] J. Daugman, "The Importance of Being Random: Statistical Principles of Iris Recognition," Pattern Recognition, Vol. 36, No. 2, 2003, pp. 279-291. doi:10.1016/S0031-3203(02)00030-4

[11] S. Ziauddin and M. N. Dailey, "Robust Iris Verification for Key Management," Pattern Recognition Letters, Vol. 31, No. 9, 2009, pp. 926-935. doi:10.1016/j.patrec.2009.12.028

[12] Massachusetts Institute of Technology, "Kerberos: The Network Authentication Protocol," august 2011. http://www.web.mit.edu/kerberos/

[13] C. Tisse, L. Martin, L. Torres and M. Robert, "Person Identification Technique Using Human Iris Recognition," Proceedings of 15th International Conference on Vision Interface, Calgary, 27-29 May 2002, pp. 294-299.

[14] R. P. Wildes, "Iris Recognition: An Emerging Biometric Technology," Proceedings of the IEEE, Vol. 85, No. 9, 1997, pp. 1348-1363. doi:10.1109/5.628669

[15] S. Sanderson and J. Erbetta, "Authentication for Secure Environments Based on Iris Scanning Technology," IEEE Colloquium on Visual Biometrics, London, 2 March 2000, pp. 8/1-8/7.

[16] J. G. Daugman, "How Iris Recognition Works," IEEE Transsctions on Circuits and Systems for Video Technology, Vol. 14, No. 1, 2004, pp. 21-30. doi:10.1109/TCSVT.2003.818350

[17] J. Wayman, A. Jain, D. Maltoni and D. Maio, "Biometric Systems, Technology, Design and Performance Evaluation," Springer, London, 2005.
[18] Hyvarinen, J. Karhunen and E. Oja, "Independent Component Analysis,” John Wiley, Hoboken, 2001. doi: $10.1002 / 0471221317$

[19] J. V. Stone, "Independent Component Analysis," MIT Press, Cambridge, 2004

[20] S. Choï, A. Cichocki and S. Amari, "Adaptative Blind Signal and Image Processing: Learning Algorithms Applications," John Wiley \& Sons, Hoboken, 2002.

[21] A. Papoulis, "Probability, Random Variables, and Stochastic Processes," 3rd Edition, McGraw-Hill, Boston, 1991.

[22] T. M. Cover and J. A. Thomas, "Elements of Information Theory," Wiley, Hoboken, 1991. doi:10.1002/0471200611

[23] P. Comon, "Independent Component Analysis-A New Concept?" Signal Processing, Vol. 36, No. 3, 1994, pp. $287-$ 314. doi:10.1016/0165-1684(94)90029-9

[24] S. Amari, "Natural Gradient for Over- and Under-Complete Bases in ICA," Neural Computation, Vol. 11, No. 8, 1999, pp. 1875-1883. doi:10.1162/089976699300015990

[25] National Institute of Standards and Technology (NIST), “Advanced Encryption Standard," NIST, FIPS PUB 197, US Department of Commerce, 2001. http://csrc.nist.gov/publications/fips/fips197/fips-197.pdf

[26] M. Dworkin, "Recommendation for Block Cipher Modes and Operations," NIST Special Publication 800-38A, 2001.

[27] C. Berrou, "Codes et Turbo Codes," Springer-Verlag, Paris, 2007. ISBN 13: 978-2-287-32739-1

[28] J. Proakis and M. Salehi, "Digital Communications," 5th Edition, McGraw-Hill, Boston, 2007. ISBN-13: 978-0072957167

[29] S. H. Moi, P. Saad, N. A. Rahim and S. Ibrahim, "Error Correction on IRIS Biometric Template Using Reed Solomon Codes," IEEE 4th Inernationnal Conference on Mathematical/Analytical Modelling and Computer Simulation (AMS), Kota Kinabalu, 26-28 May 2010, pp. 209-214. doi10.1109/AMS.2010.50

[30] Download the Application form at the Website: http://www.cbsr.ia.ac.cn/IrisDatabase.html

[31] J. Daugman, "High Confidence Visual Recognition of Persons by a Test of Statistical Independence," IEEE Transactions on Pattern Analysis and Machine Intelligence, Vol. 15, No. 11, 1993, pp. 1148-1161. doi:10.1109/34.244676

[32] L. Ma, T. Tan, Y. Wang and D. Zhang, "Efficient Iris Recognition by Characterizing Key Local Variations," IEEE Transactions on Image Processing, Vol. 13, No. 6, 2004, pp. 739-750. doi:10.1109/TIP.2004.827237

[33] L. Ma, T. Tan, Y. Wang and D. Zhang, "Personal identification based on iris texture analysis," IEEE Transactions on Pattern Analysis and Machine Intelligence, Vol. 25, No. 12, 2003, pp. 1519-1533. doi:10.1109/TPAMI.2003.1251145

[34] C. E. Shannon, "Communication Theory of Secrecy Systems," Bell System Technical Journal, Vol. 28, No. 4, 1949, pp. 656-715. 